\title{
Nota corta \\ Estudio comparativo del potencial de licuación de suelos usando las normas españolas y el Eurocódigo
}

\author{
José Luis Pastor, Roberto Tomás, Miguel Cano, Adrián J. Riquelme
}

José Luis Pastor

joseluis.pastor@ua.es

Roberto Tomás

Miguel Cano

Adrián J. Riquelme

Departamento de Ingeniería Civil. Universidad de Alicante, Apdo. Correos 99, 03080,

Alicante, España.
BOL. SOC. GEOL. MEX 2018

VOL. 70 NO. 3

P. $761-778$

http://dx.doi.org/10.18268/BSGM2018v70n3a9

\section{RESUMEN}

La licuación es un proceso por el que una masa de suelo saturado, generalmente granular y de baja compacidad, disminuye drásticamente su resistencia por un aumento de su presión de poro, habitualmente asociado a cargas monotónicas o cíclicas, tales como las inducidas por los eventos sísmicos. Este efecto es recogido tanto por diversas normativas españolas como por el Anejo Nacional Español del Eurocódigo. En este trabajo se realiza un estudio comparativo sobre las diferencias y similitudes en la metodología de comprobación del potencial de licuación empleado en las diferentes normativas. Asimismo, con objeto de cuantificar las diferencias observadas, se ha determinado el factor de seguridad frente a la licuación para tres casos reales de suelos de la provincia de Alicante. En función de los resultados obtenidos se puede concluir que existen diferencias, en algunos casos significativas, en el factor de seguridad obtenido.

Palabras clave: Licuación, NGSE-02, NCSP-07, ROM, Eurocódigo.

\section{ABSTRACT}

Liquefaction is an effect for which a saturated soil suffers a severe loss of shear strength due to an increase of pore-water pressure. This effect, which generally affects loose granular soils, is usually produced by cyclic loading such as that the induced by earthquakes. In Spain, three different national standards plus Eurocode can currently be applied in the assessment of potential liquefaction of a soil site. In this research, a comparative study of the similarities and differences between these standards has been performed. Moreover, to improve understanding of the differences between standards, the factor of safety against liquefaction has been obtained for three real cases in the province of Alicante. According to the obtained results, it can be concluded that the safety factor can present significant differences depending on the code used

Keywords: Liquefaction, NCSE02, NCSP-07, ROM, Eurocode. 


\section{Introducción}

La licuación (o licuefacción) de suelos incluye todos los procesos que conducen a una pérdida de resistencia o al desarrollo de deformaciones excesivas como resultado de una perturbación transitoria o repetida en suelos saturados no cohesivos (National Research Committee on Earthquake Engineering, 1985).

Las estructuras cimentadas sobre un terreno que sufre licuación pueden experimentar grandes asientos (Seed et al., 2003; Hamada, 2014), lo que puede llevar a las capas del suelo licuadas a comportarse como un fluido denso cuando la resistencia al corte del mismo se anula completamente. De la misma forma, los taludes y laderas también pueden verse afectados por este proceso, ocasionando su rotura y desestabilización (Seed et al, 2003; Hamada, 2014); también pueden verse afectadas las zonas planas donde haya un mínimo de efecto de borde libre, como el introducido por erosión debida a un curso de agua, fenómeno que se conoce como lateral spread (Audemard y Santis, 1991; Audemard, 2002;).

La susceptibilidad a la licuación puede evaluarse siguiendo diversos métodos (Blázquez, 2001), siendo algunos de los más utilizados el de Seed e Idriss (1971), el de Youd e Idriss (2001) y el de Yegian y Whitman (1978). Todos ellos comparan la resistencia cíclica del suelo a la licuación, obtenida mediante correlaciones con ensayos de campo (i.e. ensayo de penetración estándar SPT, ensayo de penetración con cono CPT y velocidad de onda de corte) con la solicitación cíclica que se puede producir a causa del evento sísmico.

España es un país con una sismicidad moderada (Alfaro et al., 2001; Sánchez, 2011; Perepérez, 2014), por lo que se han desarrollado normas de construcción sismorresistentes. Entre los efectos de los sismos que se deben tener en cuenta están los debidos a la licuación: "Especialmente habrá de analizarse la posibilidad de licuación (o licuefacción) de los suelos susceptibles a la misma"1 (Ministerio de Fomento, 2002), siendo obligada dicha comprobación cuando existan suelos granulares con o sin finos por debajo del nivel freático. Además, este proceso ha sido documentado en eventos sísmicos pasados (Alfaro et al., 2001; Sánchez, 2011).

En la actualidad existen en España varias normas o recomendaciones que tratan el proceso de la licuación y establecen un procedimiento para comprobar su peligrosidad durante un evento sísmico. Estas normas son la Norma de Construcción Sismorresitente: Parte General y Edificación NCSE-02 (Ministerio de Fomento, 2002) y Puentes (Ministerio de Fomento, 2007), las Recomendaciones Geotécnicas para Obras Marítimas y Portuarias ROM0.5-05 (Ministerio de Fomento, 2005) y el Eurocódigo 8: Proyecto de estructuras sismorresistentes. Parte 5: Cimentaciones, estructuras de contención y aspectos geotécnicos (AENOR, 2011). Se utiliza esta última norma debido a que el Eurocódigo 7, Proyecto geotécnico (AENOR, 2016), indica que las vibraciones debidas a terremotos se considerarán según el Eurocódigo 8 (AENOR, 2011). Por su parte, la Guía de Cimentación de Obras de Carretera (Ministerio de Fomento, 2009) y el Código Técnico de la Edificación DBSE-C (Ministerio de la Vivienda, 2008), establecen la necesidad de realizar la comprobación de que se produzca licuación del suelo remitiendo a la NCSE (Ministerio de Fomento, 2002).

Debido al alto riesgo de licuación, y a la importancia de sus efectos en determinadas zonas del continente europeo, desde la redacción del Eurocódigo 8 (AENOR, 2011) se han realizado diferentes estudios y comunicaciones (Vukobratovic y Ladinovic, 2013) para clarificar el procedimiento simplificado descrito en esta norma.

En este trabajo se realiza un estudio comparativo del cálculo del potencial de licuación mediante las cuatro normas indicadas anteriormente, efec- 
tuando un análisis de las diferencias y similitudes encontradas. Para ello, se ha uniformizado la nomenclatura de la formulación proporcionada por las diferentes normas. Por último, con objeto de cuantificar estas diferencias, se ha realizado el análisis comparativo del potencial de licuación en tres emplazamientos de la provincia de Alicante, sureste de España, del que se disponen resultados de ensayos de campo y de laboratorio.

\section{Procedimiento de cálculo del potencial de licuación adoptado por las diferentes normativas}

\subsection{NCSE-02}

Según la Norma de Construcción Sismorresistente Parte General y Edificación NCSE-02 (Ministerio de Fomento, 2002), el estudio de la seguridad frente a la licuación se puede realizar comparando la tensión tangencial equivalente del terremoto, $\tau_{E}$, con la resistencia del terreno a la licuación, $R_{L}$. A este efecto, se indica que se podrán emplear los procedimientos habituales de la ingeniería geotécnica - sísmica o el procedimiento simplificado basado en el ensayo SPT descrito en la propia norma. Este procedimiento es válido para terrenos con superficie horizontal y se deberá aplicar a todas las capas potencialmente licuables.

Esta normativa considera que existe suficiente seguridad frente la licuación si se verifica:

$$
\tau_{E} \leq R_{L} / 1,5
$$

Siendo

$\tau_{E}=$ tensión tangencial equivalente del terremoto.

$R_{L}=$ resistencia del terreno a la licuación.

$\tau_{E}$ viene definida en la Norma NCSE-02 mediante la siguiente expresión:

$$
\tau_{E}=0,65 r_{d} \sigma_{v}(a / g)
$$

O expresada de forma adimensional como la tensión de corte cíclica (CSR):

$$
C S R=0,65\left(\sigma_{v} / \sigma_{v}^{\prime}\right) \cdot(a / g) \cdot r_{d}
$$

donde:

[4]

$$
r_{d}=1-0,015 z
$$

siendo

$z=$ profundidad en metros.

$\sigma_{v} y \sigma_{v}^{\prime}=$ tensiones efectiva y total vertical a la que se comprueba la licuación.

$a=$ aceleración sísmica de cálculo.

$g$ aceleración de la gravedad.

Por su parte, la resistencia del terreno a la licuación $\left(R_{L}\right)$ se obtiene mediante la expresión siguiente:

$$
R_{L}=K_{M} \cdot C R R \cdot \sigma^{\prime}
$$

O de forma adimensional a través del valor de resistencia a la licuación (CRR) como:

$$
\left(R_{L} / \sigma^{\prime}\right)=K_{M} \cdot C R R
$$

Donde

[7]

$$
K_{M}=1,5-1,8(K-1)^{1 / 2}
$$

siendo

$K$ coeficiente de contribución. Los valores de $K$ están comprendidos entre 1,0 (puntos en que prácticamente toda la contribución a la peligrosidad sísmica procede de terremotos continentales o áreas marítimas adyacentes) y 1,3 (puntos en los que la peligrosidad sísmica procede de terremotos de la región de la falla Azores-Gibraltar).

CRR valor de resistencia a la licuación. Cociente de tensiones (Figura 1) que causa licuación en arenas limpias y limosas, y el golpeo SPT normalizado para una energía útil del $60 \%$ de la nominal y a una presión efectiva vertical de $100 \mathrm{kPa}$, $\mathrm{N}(60)$, definido según se indica a continuación. 


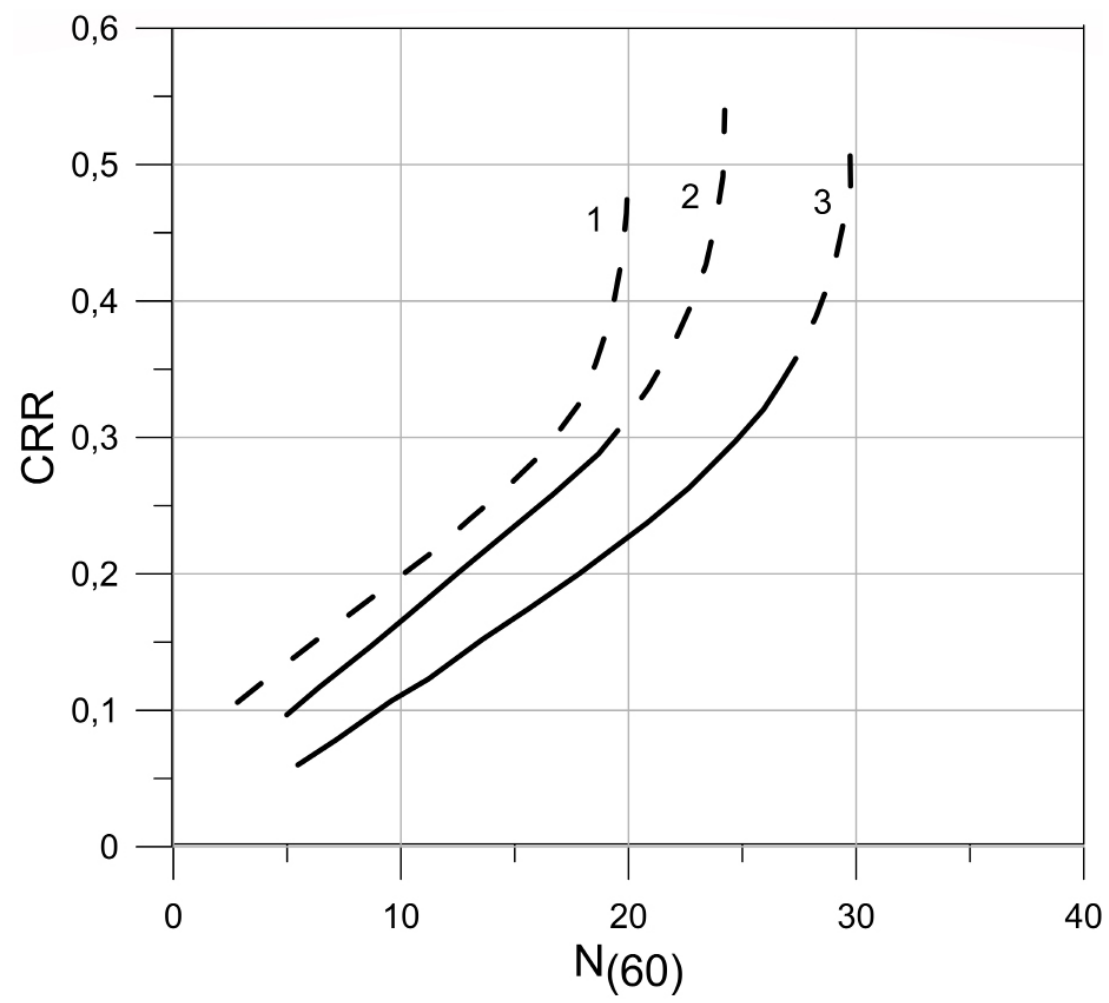

Contenido de finos: $1: 35 \% \quad 2: 15 \% \quad 3: \leq 5 \%$

Figura 1 Relación entre el cociente de tensiones que causa licuación en arenas limpias y limosas y el golpeo corregido en el SPT, N(60). NCSE-02 (Ministerio de Fomento, 2002)

[8]

$$
\mathcal{N}_{60}=\mathcal{N}\left(E_{R} / 60\right)\left(1 / \sigma_{0}^{\prime}\right)^{1 / 2}
$$

Donde

$\mathcal{N}=$ golpeo del ensayo SPT.

$E_{R}=$ porcentaje de energía en el ensayo SPT que llega realmente al terreno.

$\sigma_{v}^{\prime}=$ tensión efectiva vertical, en $\mathrm{kp} / \mathrm{cm}^{2}$, a la profundidad $z$.

\subsection{NGSP-07}

En la parte de puentes de la Norma de Construcción Sismorresistente NCSP-07 (Ministerio de Fomento, 2007) se realiza la comprobación frente a la licuación de forma análoga a la realizada para la parte general. Sin embargo, existen diferencias que es necesario resaltar.

En primer lugar, el coeficiente de seguridad frente a la licuación indicado en esta norma es de 1,5 para terrenos de cimentación y 2,0 para rellenos de trasdós de estribos.

El valor de CSR tiene la misma expresión que la indicada en [3], aunque el factor de profundidad $\left(r_{d}\right)$ tiene una expresión bilineal:

[9]

$$
r_{d}=1,0-0,0765 z \mathrm{si} \quad z \leq 9 \mathrm{~m}
$$

$$
r_{d}=1,174-0,0267 z \text { si } 9 \mathrm{~m} \leq z \leq 20 \mathrm{~m}
$$

Tabla 1. Factor de corrección del índice SPT por la longitud del varillaje (Ministerio de Fomento, 2007).

\begin{tabular}{|c|c|}
\hline $\begin{array}{c}\text { Longitud de las } \\
\text { varillas (m) }\end{array}$ & $\mathbf{C}_{\mathbf{L}}$ \\
\hline$<3$ & 0.75 \\
\hline a 4 & 0.8 \\
4 a 6 & 0.85 \\
6 a 10 & 0.95 \\
$>10$ & 1 \\
\hline
\end{tabular}


Tabla 2. Factor de corrección del índice SPT por la longitud del varillaje (Ministerio de Fomento, 2007).

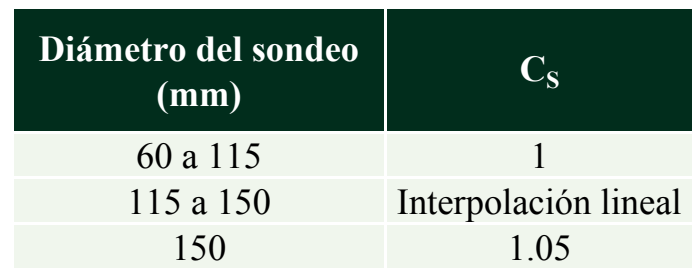

siendo $z$ la profundidad en metros.

La resistencia del terreno a la licuación se obtiene mediante la misma expresión [5], aunque la corrección del SPT cuenta con dos factores más que en la parte general y de edificación de la norma. Un factor $\mathrm{C}_{\mathrm{L}}$ en función de la longitud del varillaje (Tabla 1) y un factor $\mathrm{C}_{\mathrm{s}}$ en función del diámetro del sondeo (Tabla 2).

\subsection{ROM 0.5-05}

En las Recomendaciones Geotécnicas para Obras Marítimas y Portuarias (Ministerio de Fomento, 2005) se hace una revisión extensa del fenómeno de licuación debido a la acción sísmica. En primer lugar, además de una pequeña introducción histórica, indica que se debe realizar un reconocimiento detallado del terreno que permita obtener de forma precisa la densidad seca del suelo, la humedad, el peso específico de las partículas, la granulometría, la plasticidad y el índice $\mathrm{N}$ del ensayo SPT. Se indica también que la calidad del estudio aumenta al disponer de ensayos geofísicos que permitan obtener la velocidad de propagación de las ondas de corte en profundidad. Además, la determinación del potencial de licuación será más precisa si se realizan ensayos dinámicos del suelo en laboratorio.

La verificación de la seguridad frente a la licuación se determina, según esta norma, obteniendo el factor de seguridad:

$$
F=C R R / C S R
$$

lo que indica que cuando el valor del factor de seguridad sea inferior a 1,1 las posibilidades de licuación son altas.

En este caso, GRR se obtiene en función del valor medio del índice $\mathrm{N}$ del ensayo SPT una vez que se ha corregido por el efecto de la sobrecarga y por el efecto de la energía de ensayo. El valor corregido, $(\mathbf{N})_{60}$, se obtiene a partir de dos factores de corrección, uno en función de la sobrecarga efectiva de las tierras y otro en función de la energía que se transmite a través del varillaje a la cuchara del SPT.

El factor de corrección por la sobrecarga efectiva viene dado por la expresión:

$$
\mathcal{N}_{\text {(corregido) }}=f: \mathcal{N}
$$

Donde $\mathrm{N}$ es el valor del ensayo SPT, adoptándose un valor máximo de $50, \mathrm{y} f$ se encuentra tabulado en la Norma, incluyéndose los valores en la Tabla 3.

La corrección debida a la energía que se transmite a través del varillaje se realiza en función de la siguiente expresión:

$$
\mathcal{N}_{60}=\mathcal{N} \cdot E_{R} / 60
$$

Donde $E_{R}$ se corresponde con la energía que se transmite a través del varillaje, pudiéndose adoptar un valor del $60 \%$ a falta de información específica. De este modo, el valor queda corregido como:

Tabla 3. Factor de corrección del índice SPT por la sobrecarga efectiva de las tierras según la ROM 0.5-05 (Ministerio de Fomento, 2005).

\begin{tabular}{|c|c|}
\hline $\begin{array}{c}\text { Presión vertical efectiva al } \\
\text { nivel del ensayo }(\mathbf{k P a})\end{array}$ & Factor de corrección, $\boldsymbol{f}$ \\
\hline 0 & 2 \\
25 & 1.5 \\
50 & 1.2 \\
100 & 1 \\
200 & 0.8 \\
$\geq 400$ & 0.5 \\
\hline
\end{tabular}




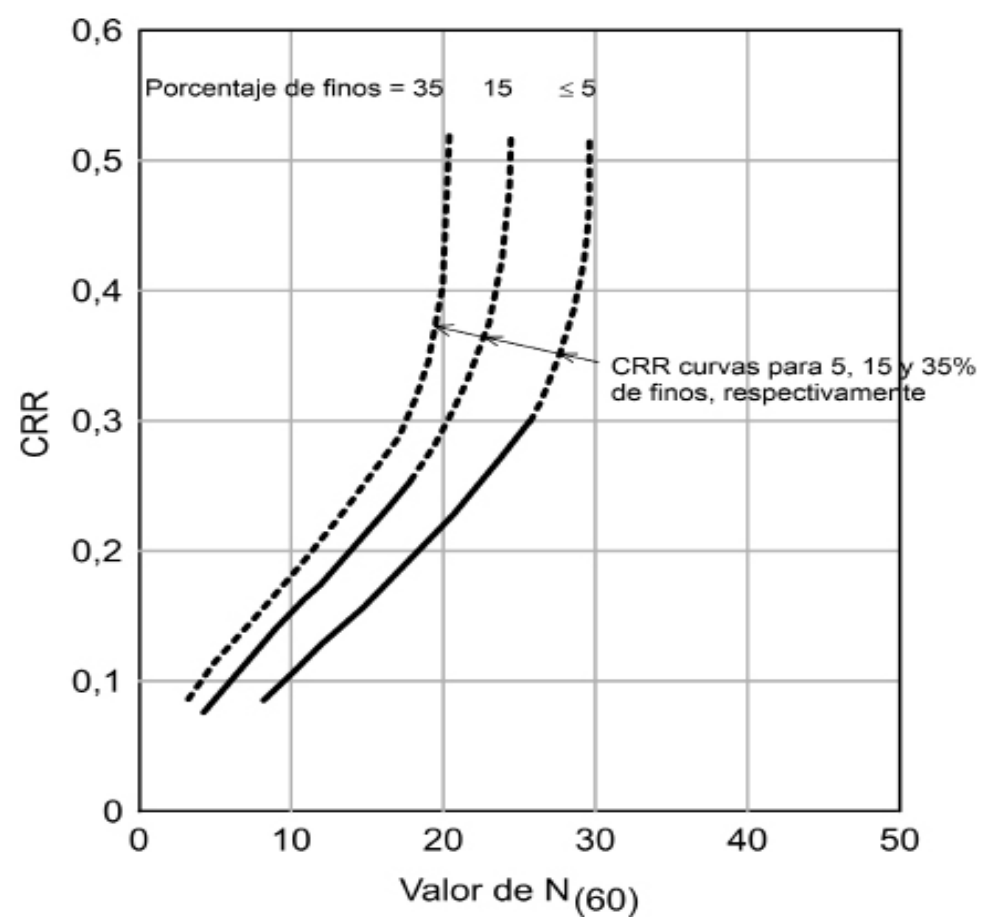

Figura 2 Valor de la resistencia a la licuación. ROM 0.5-05 (Ministerio de Fomento, 2005)

[13]

$$
\mathcal{N}_{(60)}=f \cdot \mathcal{N} \cdot\left(E_{R} / 60\right)
$$

Por otro lado, CRR viene expresada tanto en forma gráfica (Figura 2) como en una tabla en función del porcentaje de finos del suelo (Tabla 4). La misma información se puede observar de forma gráfica en la Figura 2, aunque se ha comprobado que estos valores son ligeramente diferentes a los indicados en la Tabla 4.

Los valores anteriormente indicados están obtenidos para terremotos de magnitud (M) igual a 7,5. Cuando el terremoto es de una magnitud diferente deben utilizarse los factores de corrección indicados en la Tabla 5.

El valor de CRR se debe comparar con la solicitación sísmica (CSR), definida según la expresión siguiente:

$[14]$

$$
C S R=0,65\left(\sigma_{v} / \sigma_{v}^{\prime}\right)(a / g) r_{d}
$$

siendo:

$\sigma$ tensión vertical total.

$\sigma_{v}^{\prime}$ tensión vertical efectiva.

a valor de cálculo de la aceleración horizontal máxima del terreno, equivalente a la aceleración sísmica de cálculo obtenida a partir de la aceleración sísmica básica ab, según NCSR-02.

$g$ aceleración de la gravedad.

Tabla 4. Valores del parámetro CRR según la ROM 0.5-05 (Ministerio de Fomento, 2005).

\begin{tabular}{|c|c|c|c|}
\hline & \multicolumn{3}{|c|}{ Porcentaje de finos } \\
\hline$(\mathrm{N})_{60}$ & $\leq 5 \%$ & $15 \%$ & $30 \%$ \\
\hline 5 & 0.06 & 0.1 & 0.13 \\
\hline 10 & 0.11 & 0.16 & 0.18 \\
\hline 15 & 0.16 & 0.23 & 0.26 \\
\hline 20 & 0.22 & 0.29 & 0.4 \\
\hline 25 & 0.29 & $>0.40$ & $>0.50$ \\
\hline 30 & $>0.40$ & & \\
\hline
\end{tabular}


Tabla 5. Factor de corrección del parámetro CRR según la magnitud del sismo según la ROM 0.5-05 (Ministerio de Fomento, 2005).

\begin{tabular}{|c|c|}
\hline Magnitud M & Factor de corrección \\
\hline 5.5 & 2.20 a 2.80 \\
\hline 6 & 1.76 a 2.10 \\
\hline 6.5 & 1.44 a 1.60 \\
\hline 7 & 1.19 a 1.25 \\
\hline 7.5 & 1 \\
\hline 8 & 0.84 \\
\hline 8.5 & 0.72 \\
\hline
\end{tabular}

$r_{d}$ factor de reducción dependiente de la profundidad:

$$
r_{d}=1-0,001 z^{2}
$$

siendo $z$ la profundidad a la que se realiza la comprobación, en m.

\subsection{UNE-EN 1998-5. EG-8}

El Eurocódigo 8 (AENOR, 2011) establece que se deberá realizar la comprobación de la licuación del terreno cuando esté formado por arena suelta, bien sea en amplias capas o en lentejones aislados, cuando se sitúe por debajo del nivel freático tanto si presenta finos (limos y/o arcillas) como si no.

Para poder realizar estas comprobaciones se deberá contar, al menos, con ensayos de penetración estándar (SPT) y con el análisis granulométrico del suelo.

Se podrá evitar la comprobación de licuación para cimentaciones poco profundas cuando la capa arenosa saturada se encuentre a una profundidad superior a $15 \mathrm{~m}$ desde la superficie del terreno. También cuando se cumplan las dos condiciones siguientes:

1) $a \cdot S<0,15$ siendo:

a cociente entre el valor de cálculo de la aceleración para un terreno tipo A (roca o formación geológica similar con velocidad media de la onda de corte superior a $800 \mathrm{~m} / \mathrm{s}), a_{\mathrm{g}}$, y la aceleración de la gravedad, $g$.

$S$ coeficiente dependiente del tipo de suelo. Según la propuesta del anejo nacional al Eurocódigo 8 (Industrial, s.f.), este parámetro se corresponderá con lo indicado en la NCSE-02.

2) y se cumpla, al menos, una de las condiciones siguientes:

- El contenido de arcillas de la arena es mayor del 20\% con un índice de plasticidad (IP) mayor de 10 .

- El contenido de limo de la arena es superior al $35 \%$ y el número de golpes SPT normalizado $\mathrm{N}_{(60)}$ es mayor de 20.

- Arena limpia con un número de golpes SPT normalizado $\mathrm{N}_{(60)}$ mayor de 30 .

En el resto de casos se realizará la comprobación frente a la licuación mediante correlaciones entre las mediciones de los ensayos realizados in situ y las tensiones tangenciales cíclicas críticas que causaron licuación en terremotos anteriores.

La corrección del valor del ensayo SPT es análoga a la utilizada en la NCSE-02, si bien se indica que para profundidades inferiores a $3 \mathrm{~m}$ los valores medidos en el ensayo SPT se deberán reducir un $25 \%$, y que el valor de la corrección por $\sigma^{\prime}$ debe estar comprendido entre 0,5 y 2,0 .

En el EC-8 se incluyen también unas curvas empíricas que son correlaciones entre valores del SPT normalizado $\mathrm{N}_{(60)}$ y tensiones tangenciales cíclicas (CSR) que han producido licuación en terremotos anteriores, normalizada por la presión efectiva ini$\operatorname{cial}\left(\sigma_{V}^{\prime}\right)$. Estas curvas se incluyen en la Figura 3. 
En la propia Norma se indica que estas curvas no se pueden extrapolar hasta el origen pues por debajo de un cierto umbral de $\tau_{e}$ el suelo se comporta elásticamente, no generando incrementos de presión intersticial dinámica. Por otro lado, para terremotos de magnitud distinta a 7,5 los valores de las ordenadas de las curvas en la Figura 3 se deberán multiplicar por un coeficiente CM (Tabla 6). La tensión tangencial sísmica, $\tau$, puede estimarse a partir de la siguiente expresión:

$$
\tau_{e}=0,65 a \cdot S \cdot \sigma_{v}
$$

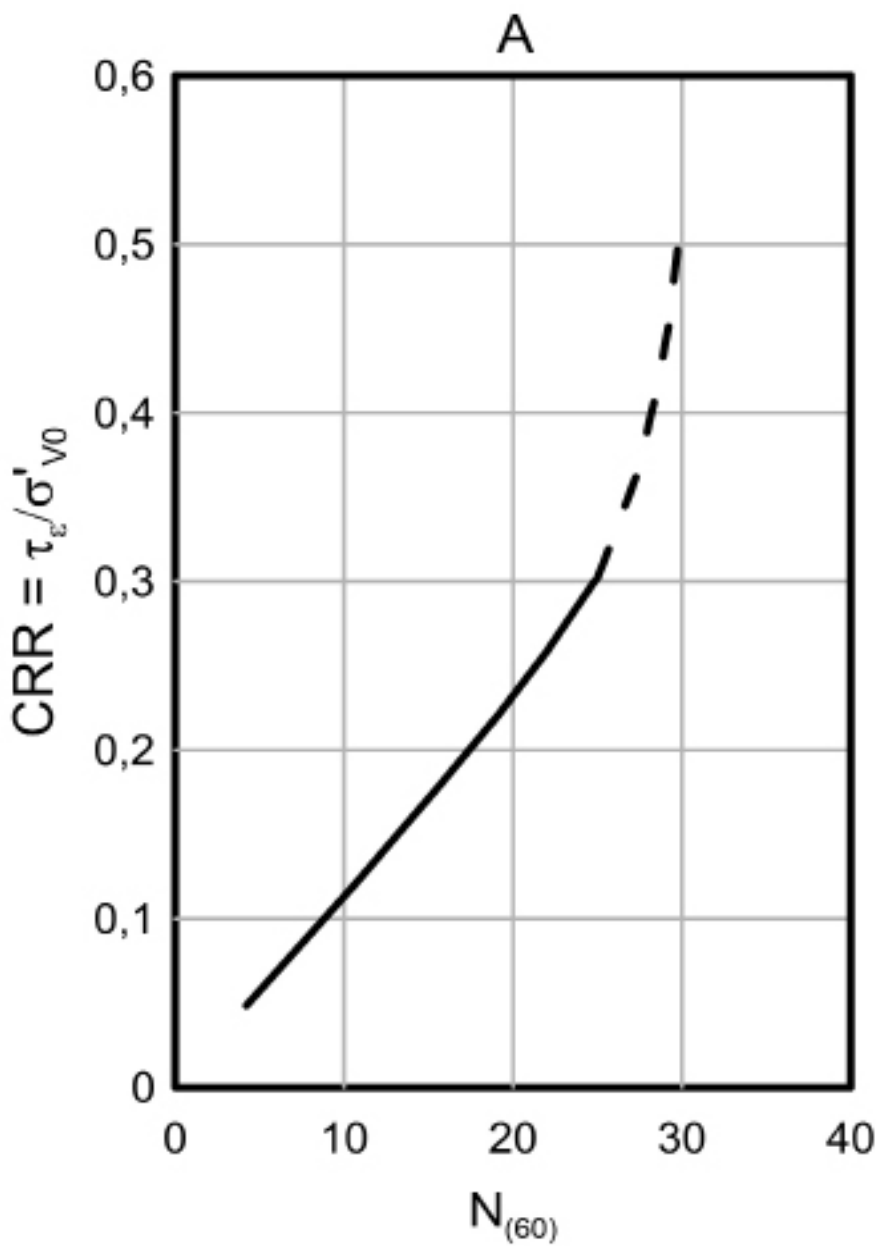
cantidad de finos inferior a 15 y 35\%, respectivamente. EC-8 (AENOR, 2011).
Donde $\sigma_{v}$ es la presión vertical total ejercida por el terreno, no pudiendo ser empleada para profundidades superiores a $20 \mathrm{~m}$, aunque según lo indicado anteriormente no es necesario realizar la comprobación para profundidades superiores a $15 \mathrm{~m}$.

Como comprobación, se considera que el terreno es susceptible a la licuación si la tensión tangencial inducida por el terremoto supera una cierta fracción $\lambda$ de la tensión crítica que se conoce ha causado licuación en terremotos anteriores. Se recomienda adoptar un valor de $\lambda=0,8$, lo que supondría un coeficiente parcial de seguridad de 1,25 .

Figura 3 Relación entre el cociente de tensiones que produce la licuación y valores de $\mathrm{N}(60)$ para terremotos de magnitud M=7,5. La gráfica A y la curva 3 de la gráfica B hacen referencia a arenas limpias (< 5\%), mientras que las curvas 2 y 1 a arenas limosas con una 
Tabla 6. Factor de corrección (CM) del parámetro CRR según la magnitud del sismo, EC-8 (AENOR, 2011).

\begin{tabular}{|c|c|}
\hline Magnitud M & CM \\
\hline 5.5 & 2.86 \\
6 & 2.2 \\
6.5 & 1.69 \\
7 & 1.3 \\
\hline 8 & 0.67 \\
\hline
\end{tabular}

\section{Análisis comparativo}

Se homogenizará la nomenclatura empleada con objeto de poder realizar la comparación entre las cuatro normativas de una forma más clara, hablando en todos los casos del valor de resistencia a la licuación (GRR), dividiendo en las normas que consideran la tensión equivalente del terremoto, $\tau_{e}$, por la tensión efectiva vertical en el punto considerado. Del mismo modo, se utilizará para la comprobación de la seguridad frente a la licuación, la tensión de corte cíclica adimensional (CSR). En este caso, se dividirá la resistencia del terreno a la licuación de la Norma NCSE-02 y NCSP-07 por la tensión efectiva vertical en el punto considerado. En la Tabla 7 se muestra la comparación de los dos parámetros indicados anteriormente, así como los factores de los que dependen estos parámetros en cada norma.

Como se observa en la Tabla 7, las expresiones de ambos parámetros son muy similares en las diferentes normas. Sin embargo, existen algunas diferencias que podrían llevar a resultados dispares. En el parámetro CSR, las normas NCSE-02, NCSP-07 y ROM 0.5-05 cuentan con un factor dependiente de la profundidad, $r_{d}$, aunque éste es diferente en cada una de ellas. Por otro lado, el parámetro CSR del EC-8 no depende de la profundidad, aunque se indica que la expresión es válida para profundidades inferiores a $20 \mathrm{~m}$, atribuible a que cualquier procedimiento simplificado ofrece resultados inciertos para profundidades superio- res a $15 \mathrm{~m}$ (Youd e Idriss, 2001). En la figura 4 se ha representado la variación de los factores de profundidad, $\mathrm{r}_{\mathrm{d}}$, para todas las normas. En dicha figura se observa cómo este factor alcanza diferencias superiores al $60 \%$ a una profundidad de 20 $\mathrm{m}$. Dado que los valores de GRR son linealmente dependientes del factor de profundidad, estos valores presentarán diferencias entre sí iguales a las mostradas por el factor de profundidad.

En las cuatro normas el parámetro CRR puede determinarse a partir de una figura en función del ensayo SPT corregido. Estas figuras son similares en todas las normas, si bien existen ligeras diferencias entre ellas. La corrección del SPT es idéntica

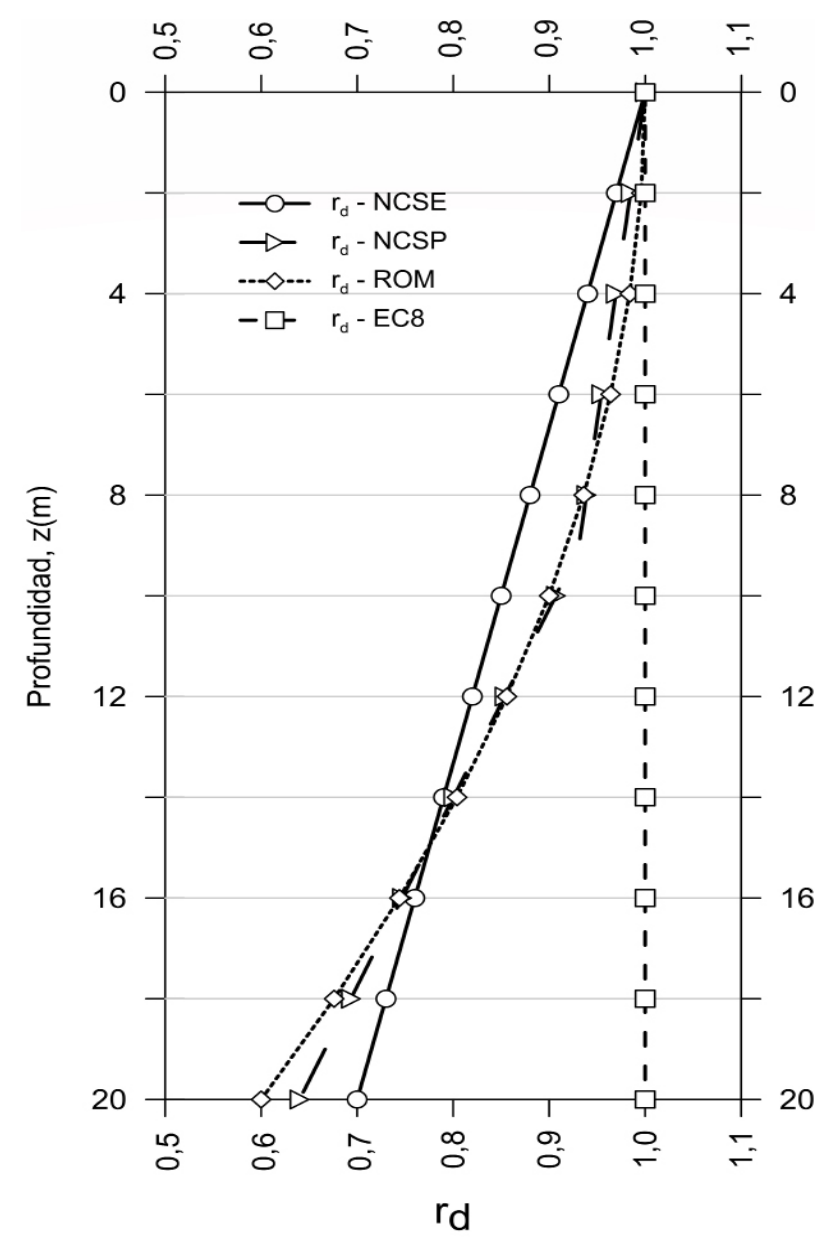

Figura 4 Valor de la resistencia a la licuación. ROM 0.5-05 (Ministerio de Fomento, 2005) 
Tabla 7. Comparación entre las diferentes normas de los valores CSR y CRR

\begin{tabular}{|c|c|c|c|c|}
\hline & NCSE-02 & NCSP-07 & ROM 0.5-05 & EC-8 \\
\hline CSR & $\begin{array}{l}\qquad 0,65 \cdot \frac{\sigma_{v}}{\sigma_{v}^{\prime}} \cdot\left(\frac{a}{g}\right) \cdot r_{d} \\
\text { Factor de profundidad: } \\
\mathrm{r}_{\mathrm{d}}=1-0,015 \mathrm{z}\end{array}$ & $\begin{array}{l}\qquad 0,65 \cdot \frac{\sigma_{v}}{\sigma_{v}^{\prime}} \cdot\left(\frac{a}{g}\right) \cdot r_{d} \\
\text { Factor de profundidad: } \\
\mathrm{r}_{\mathrm{d}}=1-0,00765 \mathrm{z} \text { si } \mathrm{z} \leq 9 \mathrm{~m} \\
\mathrm{r}_{\mathrm{d}}=1,174-0,0267 \mathrm{z} \mathrm{si} \\
\qquad 9 \mathrm{~m} \leq \mathrm{z} \leq 20 \mathrm{~m}\end{array}$ & $\begin{array}{l}\quad 0,65 \cdot \frac{\sigma_{v}}{\sigma_{v}^{\prime}} \cdot\left(\frac{a}{g}\right) \cdot r_{d} \\
\text { Factor } \\
\text { de profundidad: } \\
\mathrm{r}_{\mathrm{d}}=1-0,001 \mathrm{z}^{2}\end{array}$ & $\begin{array}{l}\qquad, 65 \cdot \frac{\sigma_{v}}{\sigma_{v}^{\prime}} \cdot\left(\frac{a}{g}\right) \\
\text { Sin factor de profundidad } \\
\text { Válido para } \mathrm{z}<20 \mathrm{~m}\end{array}$ \\
\hline CRR & $\begin{array}{l}\text { CRR directamente de gráfico } \\
\text { (Figura 1) tras corregir el SPT } \\
N_{(60)}=N \cdot\left(\frac{E_{R}}{60}\right) \cdot\left(1 / \sigma_{v}^{\prime}\right)^{1 / 2} \\
\sigma_{\mathrm{v}}^{\prime} \text { en } \mathrm{kp} / \mathrm{cm}^{2} \\
\text { Corrección de CRR mediante } \\
\text { factor } \mathrm{K}_{\mathrm{M}} \\
K_{M}=1,5-1,8(K-1)^{1 / 2} \\
\mathrm{~K} \text { coef. de contribución en } \\
\text { función de la peligrosidad } \\
\text { sísmica }\end{array}$ & $\begin{array}{l}\text { CRR directamente de gráfico (Figura 1) } \\
\text { tras corregir el SPT } \\
\qquad N_{(60)}=N \cdot C_{L} \cdot C_{S} \cdot\left(\frac{E_{R}}{60}\right) \cdot\left(1 / \sigma_{v}^{\prime}\right)^{1 / 2} \\
\sigma_{v}^{\prime} \text { en kp/cm }{ }^{2} \\
C_{\mathrm{L}} \text { factor en función de la longitud del } \\
\text { varillaje (Tabla 1). } \\
\mathrm{C}_{\mathrm{S}} \text { factor en función del diámetro del } \\
\text { sondeo (Tabla 2). } \\
\text { Corrección de CRR mediante factor } \\
\mathrm{K}_{\mathrm{M}} \\
\qquad K_{M}=1,5-1,8(K-1)^{1 / 2} \\
\text { K coef. de contribución en función de } \\
\text { la peligrosidad sísmica }\end{array}$ & $\begin{array}{l}\text { CRR directamente de } \\
\text { gráfico (Figura 2) o de } \\
\text { Tabla 4, tras corregir el } \\
\text { SPT } \\
\qquad N_{(60)}=f \cdot N \cdot \frac{E_{R}}{60} \\
\text { f factor de corrección en } \\
\text { función de la presión } \\
\text { efectiva (Tabla 3). } \\
\text { Corrección de CRR para } \\
\text { magnitud de sismo } \\
\text { M } \neq 7,5 \text { (Tabla 5). }\end{array}$ & $\begin{array}{l}\text { CRR directamente de } \\
\text { gráfico (Figura 3) tras } \\
\text { corregir el SPT } \\
N_{(60)}=N \sqrt{\frac{100 k P a}{\sigma_{V}^{\prime}}} \cdot \frac{E_{R}}{60} \\
\text { Con } 0,5 \sqrt{\frac{100 k P a}{\sigma_{V}^{\prime}}} \leq 2,0 \\
\mathrm{~N} \text { se multiplica por } 0,75 \\
\text { para } \mathrm{z}<3 \mathrm{~m} \\
\text { Corrección de CRR para } \\
\text { magnitud de sismo M } \neq 7,5 \\
\text { (Tabla 6). }\end{array}$ \\
\hline
\end{tabular}


en la NCSE-02 y EC-8, pero ligeramente diferente tanto en la NCSP-07, donde cuenta con un factor de longitud de varillaje y otro por diámetro del sondeo, como en la ROM 0.5-05 donde el factor de corrección está tabulado en función de la presión efectiva, y no es completamente concordante con la función continua indicada en las otras normas. En la Figura 5 se muestra este factor en función de la presión efectiva vertical. En todas las normas se obtiene el mismo factor de corrección para una presión de $100 \mathrm{kPa}$. Sin embargo, por ejemplo, para una presión vertical de $25 \mathrm{kPa}$ el factor obtenido para la NCSE y NCSP es un 33\% superior al obtenido mediante la ROM.

Otra diferencia entre las normas es el factor de corrección para terremotos de magnitud $(\mathbf{M})$ diferente a 7,5. Las normas ROM 0.5-05 y EC-8 cuentan con este factor tabulado (Tablas 3 y 4 , respectivamente), si bien los valores son algo diferentes entre sí. La NCSE-02 y la NCSP-07 cuentan por su parte con un factor $\mathrm{K}_{\mathrm{M}}$ en función del coeficiente de contribución, coeficiente definido en la norma según la situación geográfica considerada y que tiene en cuenta la influencia de los distintos tipos de terremotos esperados según la peligrosidad sísmica de cada zona. Este factor es equivalente al factor de corrección por magnitud del sismo de las otras dos normas y toma un valor de 1,5 para gran parte del territorio español, excepto en algunos términos municipales de Andalucía y Extremadura donde alcanza un valor mínimo de 0,51.

En la Figura 6 se ha representado el valor del factor de corrección en función de la magnitud del sismo (M), que tiene en cuenta las particularidades sísmicas de cada región. Este factor toma valores diferentes, existiendo una diferencia inferior entre los valores obtenidos mediante la ROM y el EC-8 que con los obtenidos entre éstas y la NCSE y la NCSP. Puesto que a la Península Ibérica se le atribuye un nivel máximo de magnitud momento (M) igual a 7 (Martínez-Solares, 2012, Perepérez, 2014), el análisis se centra en sismos de magnitu- des iguales o inferiores a este valor. Se observa en la Figura 6 cómo la mayor diferencia entre normas se produce para sismos de magnitud igual a 5,5, donde el factor de corrección obtenido mediante el EC-8 es superior en más de un $80 \%$ al obtenido mediante la NCSE y NCSP. Para estas normas se ha representado únicamente el valor más habitual de 1,5, si bien como se ha dicho anteriormente en algunos puntos del oeste y suroeste español (Andalucía y Extremadura) se alcanza un valor mínimo de 0,51 .

El factor de seguridad exigido o recomendado en cada Norma también es diferente, mientras que la NCSE-02 indica un factor de seguridad de 1,50, la

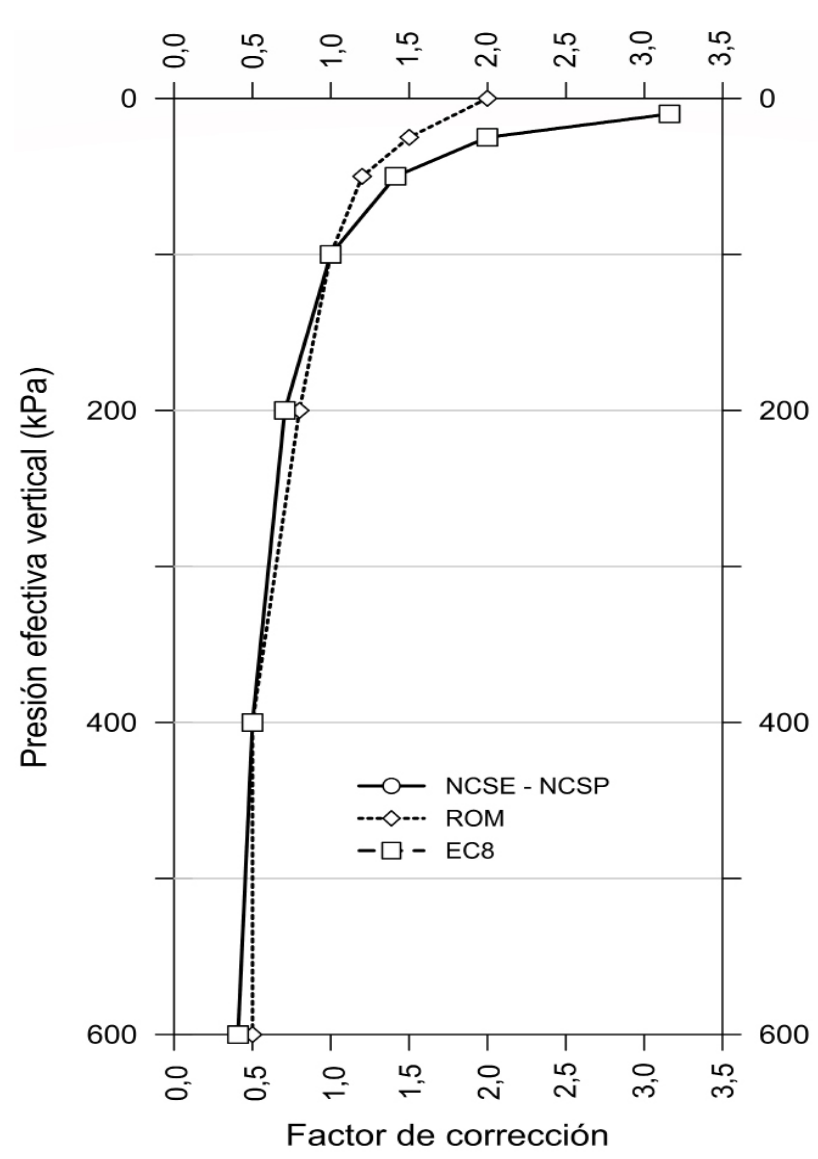

Figura 5 Factor de corrección en función de la presión efectiva vertical. 


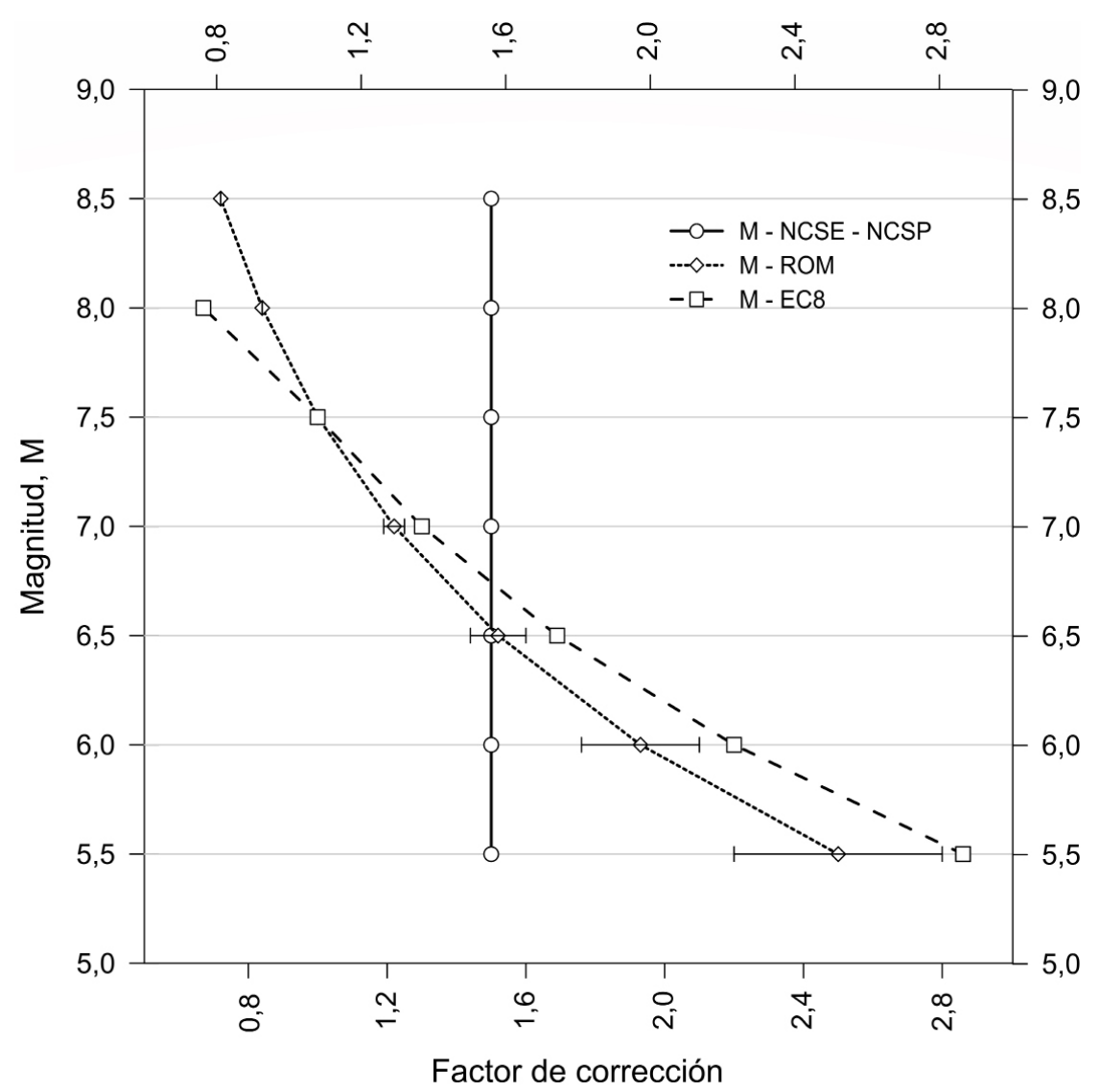

Figura 6 Factor de corrección en función de la Magnitud (M) del terremoto para las cuatro normas. En la NCSE - NCSP se ha representado el valor más habitual en el territorio nacional.

NCSP-07 refiere el mismo valor para terrenos de cimentación y 2,0 para rellenos de trasdós de estribos, recomendando el EG-8 un coeficiente parcial de seguridad de 1,25. Por su parte, la ROM 0.5-05 dice que se debe entender que las posibilidades de licuación son altas si el coeficiente de seguridad es inferior a 1,10 , sin indicar un factor de seguridad explícito.

\section{Casos de estudio}

Con objeto de evidenciar las diferencias entre las cuatro normativas estudiadas, se propone cuantificar el factor de seguridad y el potencial de licua- ción a través del estudio de tres emplazamientos reales situados en los términos municipales de Benijófar, Alicante y Santa Pola, todos ellos en la provincia de Alicante (Figura 7).

\subsection{BENIJÓFAR (ALICANTE)}

El primer emplazamiento estudiado está situado en la población de Benijófar (Alicante), con una aceleración sísmica básica $(\mathrm{ab})$ de $0,15 \mathrm{~g}$, formado por arenas limosas (contenido de finos ligeramente inferior al 30\%) y con el nivel freático a $2 \mathrm{~m}$ de profundidad. La densidad aparente del terreno situado por encima del nivel freático es de $16 \mathrm{kN} /$ $\mathrm{m}^{3}$ y la saturada del suelo por debajo del nivel 


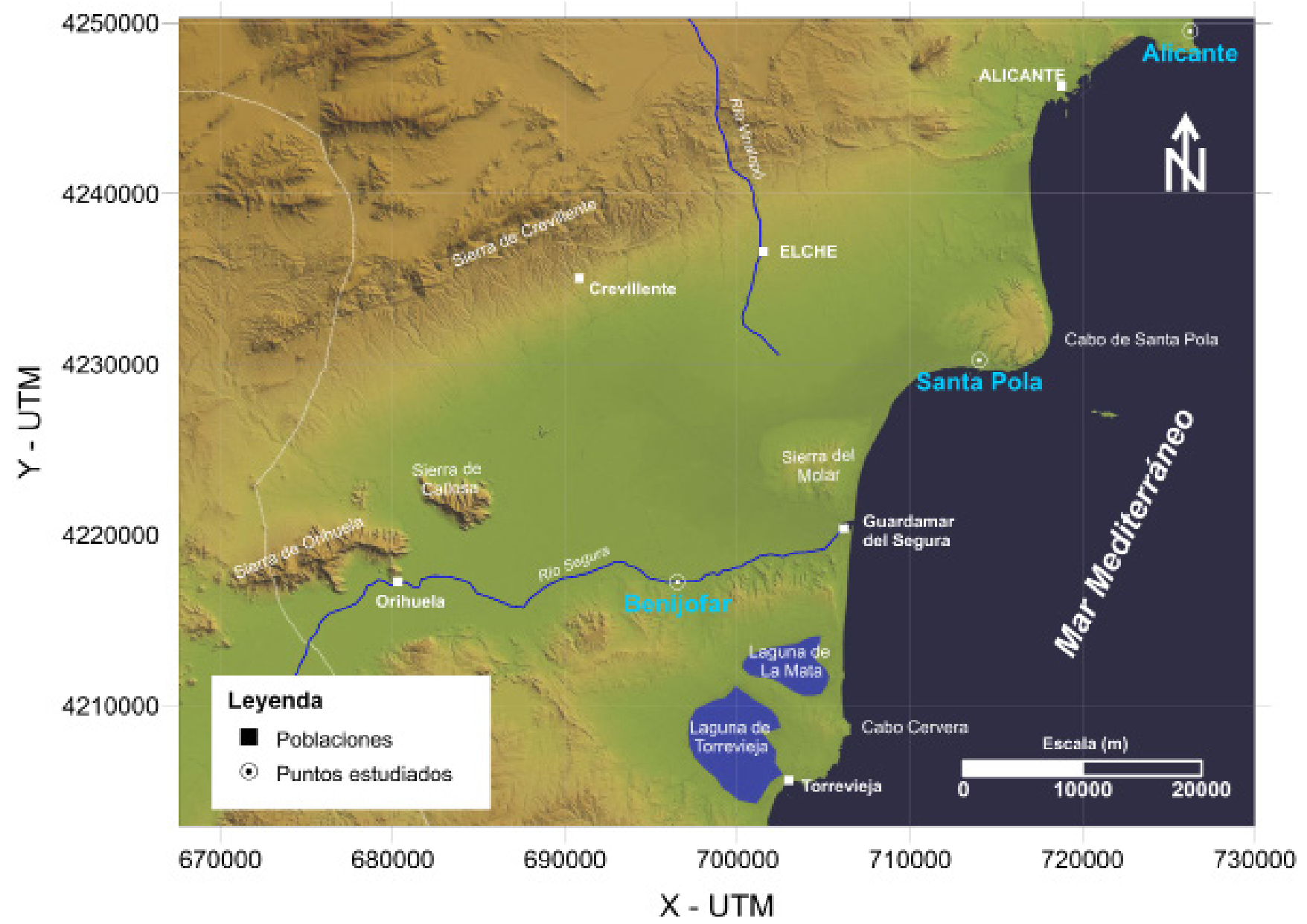

Figura 7 Situación de los puntos donde se ha evaluado el potencial de licuación. Centro del mapa (EPSG: 25830) x=706563.66, y= 4226631.38. Modificado de Terrasit (Institut Cartográfic Valencià).

freático, $19 \mathrm{kN} / \mathrm{m}^{3}$. El coeficiente $\mathrm{G}$ del terreno considerado es de 1,6 y el coeficiente de contribución, $\mathrm{K}=1$.

La columna litológica y los resultados del ensayo de penetración estándar (SPT) se muestran en la Figura 8. En la misma figura se han representado también los valores en profundidad de CSR, GRR y el factor de seguridad obtenidos como el cociente entre los dos parámetros anteriores para las tres normativas consideradas.

CSR presenta variaciones significativas entre las normativas consideradas. Por un lado, el no considerar el factor de profundidad $r_{d}$ en el EC-8 hace que el valor CSR se distancie entre esta norma y el resto conforme aumenta la profundidad. Por otro lado, el hecho de que la expresión del factor $r_{d}$ sea diferente hace que hasta $15 \mathrm{~m}$ de profundidad el valor CSR sea superior en la ROM-05, mientras que a partir de esa profundidad sea superior en la NCSE.

En lo que respecta a CRR, el valor obtenido es similar en las cuatro normas, si bien se han obtenido los valores superiores para la NCSE-02 y NCSP-07 a cualquier profundidad. Estos valores se deben al coeficiente de contribución indicado en esta norma para gran parte del territorio nacional. Por su parte, se ha considerado un sismo de magnitud 7,0 para la obtención del coeficiente de corrección para las normas ROM-05 y EG-8. Se ha utilizado un sismo de esa magnitud debido 


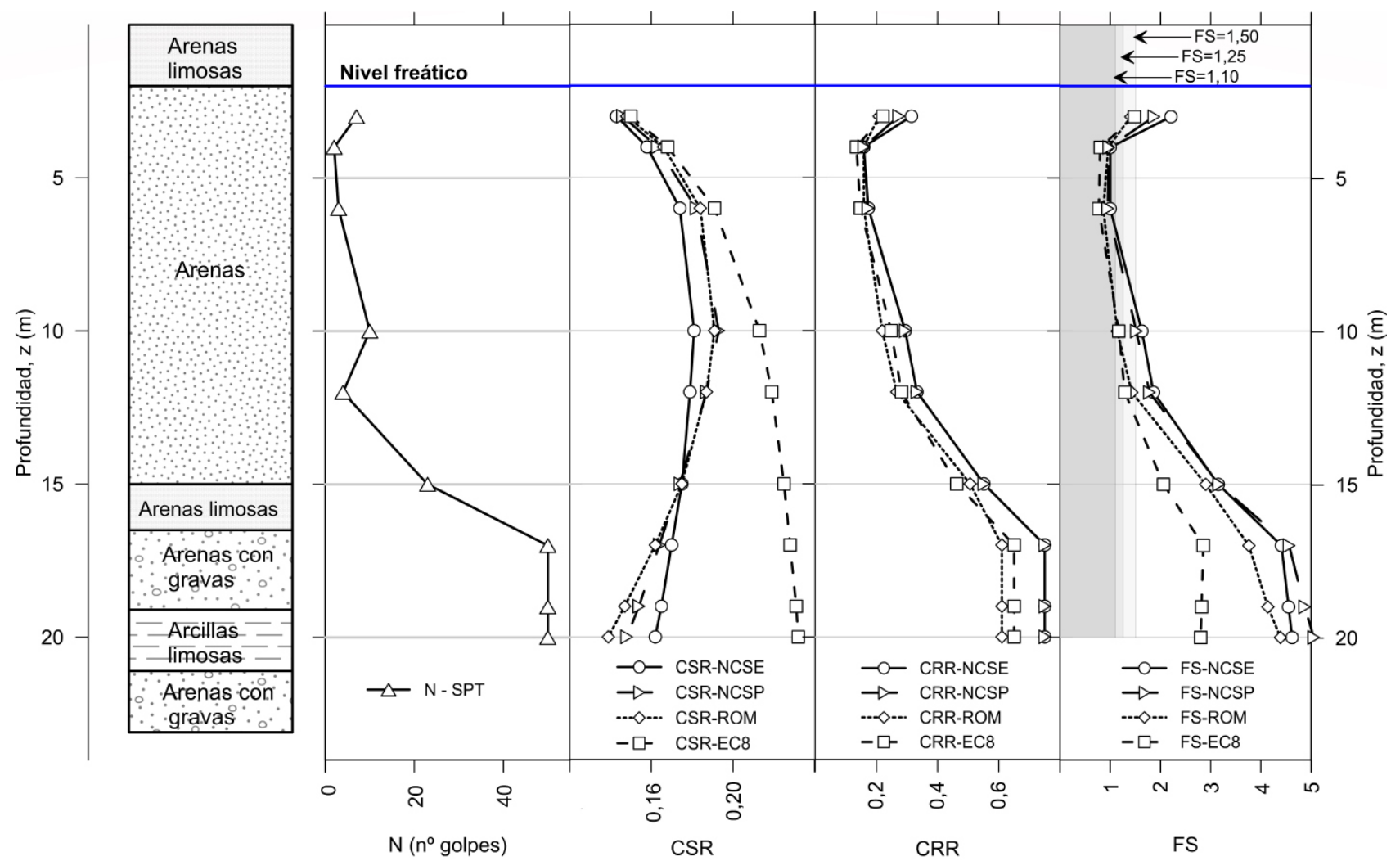

Figura 8 Valores de golpeos SPT, CSR, CRR y factor de seguridad (FS) para el ejemplo estudiado en Benijófar (Alicante).

a que en la bibliografía consultada se indica que la Península Ibérica presenta un nivel máximo de magnitud momento (M) igual a 7 (Martínez-Solares, 2012, Perepérez, 2014), magnitud muy similar a la estimada en el evento sísmico del año 1829 en Torrevieja (Alicante) (Martínez-Solares, 2012). Por último, la NCSE-02 y NCSP-07 presentan el factor de seguridad más elevado para toda la profundidad estudiada. Tanto para el EC-8 como para la ROM-05 se obtiene un factor de seguridad inferior a la unidad entre 6 y $9 \mathrm{~m}$ de profundidad. Los distintos factores de seguridad exigidos por las normas hacen que según todas ellas exista peligro de licuación entre 4 y 10 m de profundidad.

\subsection{ALICANTE}

El segundo ejemplo estudiado está situado en el Término Municipal de Alicante, con una aceleración sísmica básica (ab) de 0,14g. El suelo está for- mado por arenas limosas (contenido de finos del $30 \%$ ) y el nivel freático está a $2 \mathrm{~m}$ de profundidad. La densidad aparente del terreno es de $15 \mathrm{kN} / \mathrm{m}^{3}$ y la densidad saturada de $18 \mathrm{kN} / \mathrm{m}^{3}$. El coeficiente $\mathrm{C}$ del terreno considerado es de 1,7 y $\mathrm{K}=1$.

La columna litológica, los valores SPT y los resultados de los cálculos realizados se muestran en la Figura 9.

CSR presenta el mismo comportamiento que el mostrado en el ejemplo anterior, donde $r_{d}$, que contemplan todas las normas excepto el EC-8, hace que el valor CSR difiera conforme aumenta la profundidad entre estas normas y el EC-8.

El valor obtenido para CRR es similar en todas las normas hasta $9 \mathrm{~m}$. A partir de aquí se alcanza prácticamente el mismo valor para la ROM-05 y el EC-8, mientras que el obtenido mediante la NCSE-02 y NCSP-07 aumenta en mayor proporción, reduciéndose posteriormente a $20 \mathrm{~m}$ de profundidad. Los valores superiores obtenidos en la 
NCSE-02 y NCSP-07 a cualquier profundidad se deben al coeficiente de contribución indicado en esta norma para gran parte del territorio nacional. El factor de seguridad conseguido mediante la ROM-05 y el EC-8 es muy similar hasta una profundidad de $9 \mathrm{~m}$. A partir de ésta, el factor según la $\mathrm{ROM}$ se hace cada vez mayor, distanciándose del obtenido según el EC-8. El factor de seguridad según la NCSE y NCSP es superior al de las otras dos normas, siendo esta diferencia máxima entre 13 y $18 \mathrm{~m}$. Sin embargo, para $20 \mathrm{~m}$ de profundidad es inferior al generado según la ROM.

\subsection{SANTA POLA (ALICANTE)}

El tercer y último ejemplo estudiado está situado en la ciudad de Santa Pola (Alicante), con una aceleración sísmica básica (ab) de $0,15 \mathrm{~g}$. El suelo está formado por arenas (contenido de finos inferior al $15 \%$ ) y el nivel freático se encuentra a $0,2 \mathrm{~m}$ de profundidad. La densidad aparente del suelo es de $16 \mathrm{kN} / \mathrm{m}^{3}$ y la densidad saturada de $19 \mathrm{kN} / \mathrm{m}^{3}$. El coeficiente $\mathrm{C}$ del terreno considerado es de 1,8 y $\mathrm{K}=1$.

La columna litológica, los valores SPT y los resultados de los cálculos realizados se muestran en la Figura 10.

CSR presenta la misma tendencia a la mostrada en los ejemplos anteriores, donde el valor obtenido según el EC-8 es el mayor de los cuatro, haciéndose la diferencia mayor conforme aumenta la profundidad. La diferencia de valor entre las normas es inferior en este caso debido a que la profundidad estudiada, hasta la que se tienen datos, es únicamente de $10 \mathrm{~m}$.

En lo que respecta a CRR, se obtiene práctica-

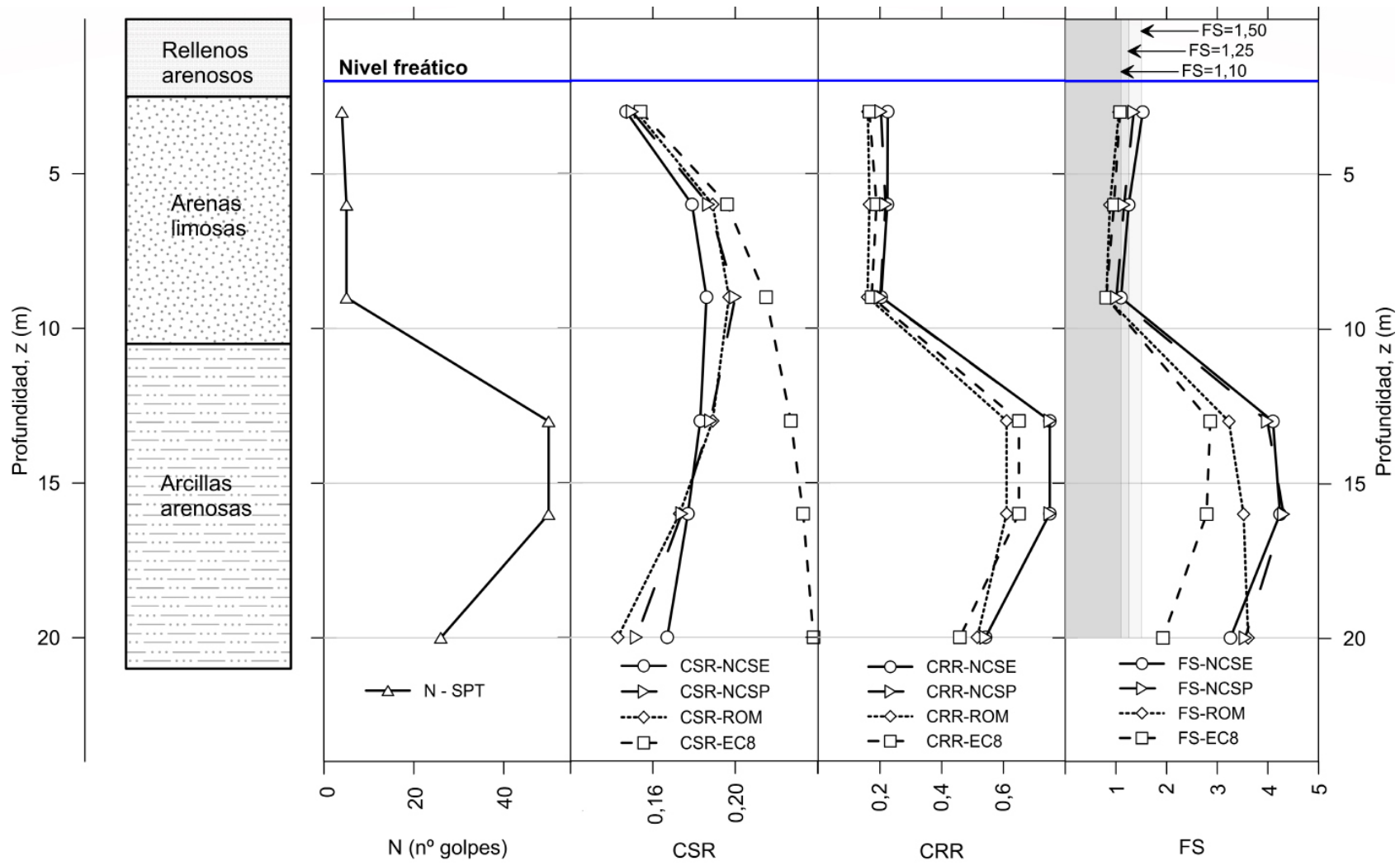

Figura 9 Valores de golpeos SPT, CSR, CRR y factor de seguridad (FS) para el ejemplo estudiado en Alicante. 


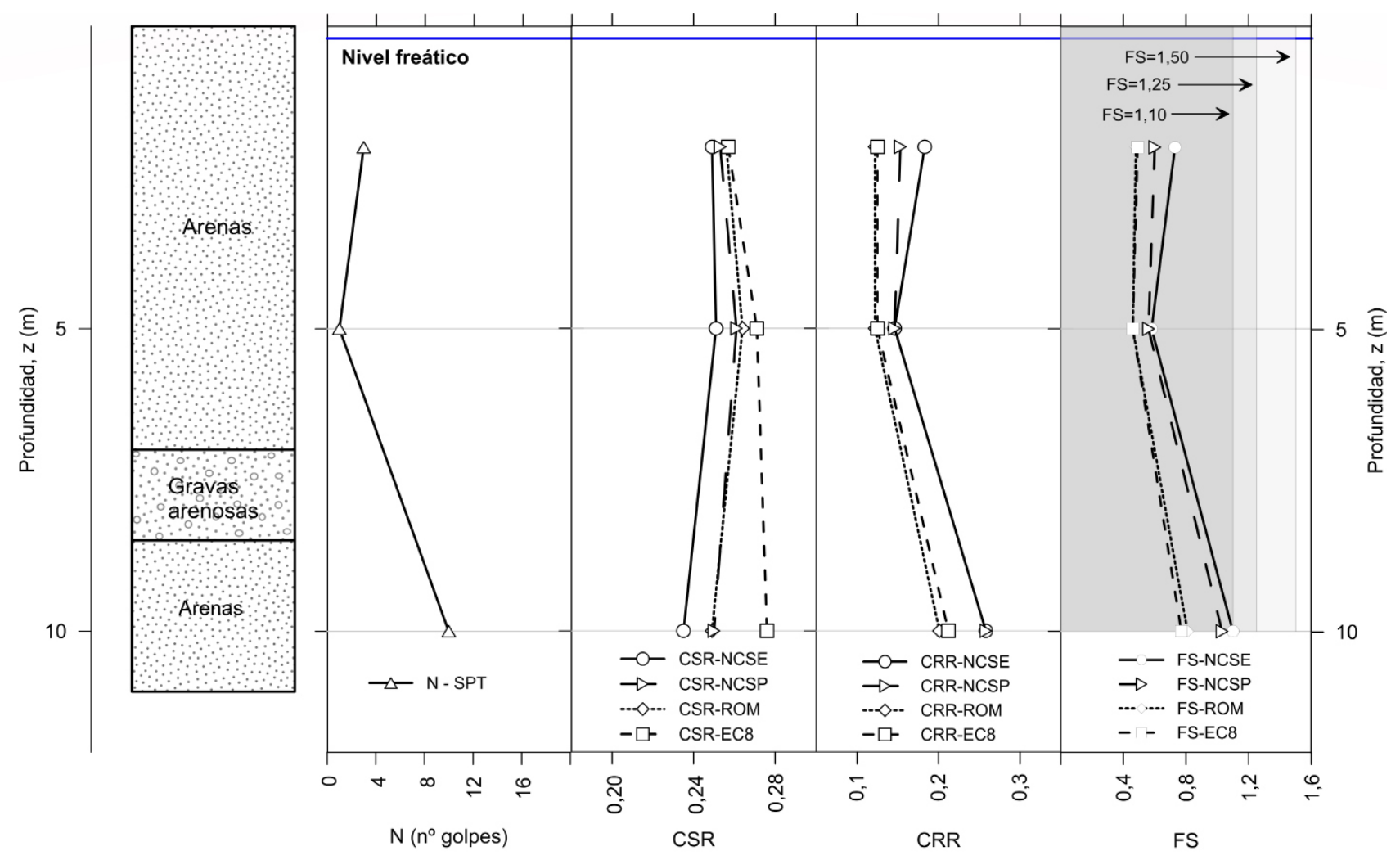

Figura 10 Valores de golpeos SPT, CSR, CRR y factor de seguridad (FS) para el ejemplo estudiado en Santa Pola (Alicante).

mente el mismo valor para la ROM-05 y el EC-8, y valores superiores en la NCSP-07 y NCSE-02.

$\mathrm{El}$ factor de seguridad es prácticamente el mismo para la ROM-05 y el EC-8, siendo el de la NCSP07 algo superior y el mayor de todos los obtenidos mediante la NCSE-02. En este ejemplo, el factor de seguridad es inferior a 1 en toda la profundidad estudiada para las normas ROM-05 y EC-8, así como hasta $5 \mathrm{~m}$ para la NCSE-02 y NCSP-07, pero a $10 \mathrm{~m}$ de profundidad se obtiene un valor ligeramente superior a 1. Existe, por tanto, un claro riesgo de licuación para este emplazamiento según todas las normas consideradas.

\section{Conclusiones}

Actualmente, existen en España tres normativas o recomendaciones diferentes, aplicables a distintos ámbitos de construcción (edificación, puentes y obras en entornos portuarios) que tratan la licuación de suelos debido al efecto de los sismos (NCSE-02, NCSP-07 y ROM 0.5-05). Además, también es de aplicación el anejo español del Eurocódigo (EC-8), que tiene un ámbito de aplicación más transversal. En todas ellas se indican procedimientos simples, basados en ensayos in situ (SPT) con gran similitud entre ellos, que determinan el potencial de licuación de un suelo.

La diferencia en la expresión del factor dependiente de la profundidad en la NCSE-02, NCSP-07 y ROM 0.5-05, así como la no evaluación de este factor por el EC-8, hacen que los valores de CSR sean diferentes entre las cuatro normas, especialmente conforme se incrementa la profundidad, incluso con diferencias de hasta el $60 \%$ en alguno de los casos estudiados.

El valor de CRR puede ser ligeramente diferente para cada una de las normas, pero tan solo por utilizar criterios diferentes para la obtención del 
factor de corrección del ensayo SPT en función de la tensión efectiva y por el factor de corrección por longitud del varillaje. Asimismo, existen diferencias por la corrección a causa de la magnitud del sismo, cuyo valor es distinto entre las normas. Las diferencias en la estimación de la tensión de corte cíclica y de la resistencia a la licuación hacen que el factor de seguridad frente a la licuación obtenido pueda variar según la norma considerada, obteniendo valores muy dispares de la seguridad frente a la licuación.

En los casos de estudio analizados, los mayores factores de seguridad se han obtenido para la NCSE-02 y NCSP-07, si bien estas normas exigen un factor de seguridad superior al requerido por las otras dos. Considerando como referencia la diferencia entre el factor de seguridad calculado y el exigido por la norma correspondiente, expresado en tanto por ciento, se puede concluir que entre las normas españolas, las que hacen referencia a obra civil (NCSP-07 y ROM 0.5-05) son más conservadoras que la norma aplicable a edificación (NCSE-02). Ello es atribuible, en principio, al diferente uso y vida útil de las estructuras. Por otro lado, el EC-8, de forma general, proporciona resultados más conservadores que las otras normas.

\section{Agradecimientos}

El trabajo ha sido parcialmente financiado por los proyectos del Ministerio de Economía y Competitividad de España y los Fondos Feder TIN201455413-C2-2-P y el proyecto UA GRE140-4.

\section{Referencias}

Asociación Española de Normalización y Certificación (AENOR), 2011, Eurocódigo 8: Proyecto de estructuras sismorresistentes. Parte 5: Cimentaciones, estructuras de contención y aspectos geotécnicos (UNEEN 1998-5): Madrid. Se recomienda agregar medio de publicación y número total de páginas.

Asociación Española de Normalización y Certificación (AENOR), 2016, Eurocódigo 7: Proyecto geotécnico. Parte 1: Reglas generales (UNE-EN 1997-1): Madrid.

Alfaro, P., Delgado, J., Estévez, A. y López Casado, C., 2001, Paleoliquefaction in the Bajo Segura basin (Eastern Betic Cordillera): Acta Geológica Hispánica, 36(3-4), 233-244.

Audemard, F. A., 2002, Soil Liquefaction During the Caracas 1967 and the Boca de Tocuyo 1989 Earthquakes, Venezuela: Its significance for Human Settlements on Active Alluvial and Coastal Areas, in Jackson Trevor (ed.), Caribbean Geology into the Third Millenium: Mona, University of the West Indies, 229-234.

Audemard, F. A. and Santis, F., 1991, Survey of liquefaction structures induced by recent moderate earthquakes: Bulletin of the International Association of Engineering Geology, 44(1), 5-16.

Blázquez, R., 2001, Potencial de licuefacción de suelos: Madrid, Asociación Española de Ingeniería Sísmica.

Fundación para el fomento de la innovación industrial, 2010, Propuesta del anejo nacional español a la norma europea EN 1998 (Eurocódigo 8). Estructuras resistentes al sismo. Madrid.

Hamada, M., 2014, Engineering for earthquake disaster mitigation: Tokyo, Springer Japan.

Institut Cartográfic Valencià, Terrasit (en línea), 2012, IDE de la Comunitat Valenciana, disponible en: ‘http://terrasit.gva.es/es/ ver4551’, consultado el 9 de marzo de 2017.

Instituto Universitario de Investigación Andaluz de Geofísica y Prevención de Desastres Sísmicos, Universidad de Granada (en línea), 
disponible en: 'http://iagpds.ugr.es/pages/ informacion_divulgacion/sismos_superior_ vii', consultado el 10 de marzo de 2017.

Martínez-Solares, J. M., 2012, Sismicidad preinstrumental. Los grandes terremotos históricos en España: Eseñanza de las Ciencias de la Tierra, 19 (3), 296-304.

Ministerio de Fomento, 2002, Real Decreto 997/2002 por el que se aprueba la Norma de Construcción Sismorresistente: Parte General y Edificación (NCSE-02): Madrid, Boletín Oficial del Estado, 27 del septiembre de 2002, 96 p.

Ministerio de Fomento, 2005, Recomendaciones Geotécnicas para Obras Marítimas y Portuarias (ROM 0.5-05): Madrid, Puertos del Estado, 546 p.

Ministerio de Fomento, 2007, Real Decreto $637 / 2007$, de 18 de mayo, por el que se aprueba la Norma de Construcción Sismorresistente: Puentes (NCSP-07): Madrid, Boletín Oficial del Estado, 22 de septiembre de 2007, 134 p.

Ministerio de Fomento, 2009, Guía de Cimentaciones en Obras de Carretera: Madrid, Centro de Publicaciones, 299 p.

Ministerio de la Vivienda, 2008, Texto modificado por RD 1371/2007 y corrección de errores, Código Técnico de la Edificación. Documento Básico - Seguridad Estructural Cimientos (CTE DB-SE-C): Madrid, Boletín Oficial del Estado, 19 de octubre de 2007 y corrección de errores 25 de enero de 2008, $164 \mathrm{p}$.

National Research Committee (NRG) on Earthquake Engineering, Council on
Engineering and Technical Systems, 1985, Liquefaction of soils during earthquakes, Washington, D.C..

Perepérez, B., 2014, La peligrosidad sísmica y el factor de riesgo: Informes de la Construcción, 66 no 534.

Sánchez, F. V., 2011, El terremoto de Alhama de Granada de 1884 y su impacto: Anuari Verdaguer, 0(19), 11-45.

Seed, H. B., and Idriss, I. M., 1971, Simplified procedure for evaluating soil liquefaction potential: Journal of the Soil Mechanics and Foundation Division, 97(SM9), 1249-1273.

Seed, R. B. et al., 2003. Recent advances in soil liquefaction engineering: A unified and consistent framework, University of California, Long Beach.

Vukobratovic, V., and Ladjinovic, D., 2013, Liquefaction assessment according to Eurocode 8, Proceedings of the 15th International Symposium of MASE: Struga (Macedonia), Macedonian Association of Structural Engineers.

Yegian, M.K., and Whitman, R.V, 1978, Risk analysis for earthquake-induced ground failure by liquefaction: Journal of the Geotechnical Division ASCE, 104 (GT7), 921-938.

Youd, T.L., and Idriss, I. M., 2001, Liquefaction resistance of soils: Summary report from the 1996 NGEER and 1998 NGEER/NSF workshops on evaluation of liquefaction resistance: Journal of Geotechnical and Geoenvironmental Engineering, 127 (4), 297-313. 